\title{
A bountiful harvest on Ceres
}

\author{
In its second extended mission at Ceres, the Dawn spacecraft returned a harvest of high-resolution data on the \\ intriguing Occator crater, a landmark for understanding the role of impacts in shaping ice-rich bodies, explain \\ Project Scientist Julie Castillo-Rogez and Chief Engineer Marc Rayman.
}

T he Dawn mission, NASA's ninth Discovery project, launched in 2007 and concluded operations in October 2018 after orbiting and exploring the two largest objects in the asteroid belt: protoplanet Vesta (2011-2012) and dwarf planet Ceres (2015-2018). Both bodies are considered fossils from the dawn of the Solar System, hence their exploration was motivated by the goal to constrain early Solar System history. One key result is the strong evidence for the origins of the two objects in different reservoirs. While Vesta probably formed near where it is now, Ceres probably migrated from the outer Solar System, as indicated by its high carbon and nitrogen content (M. C. De Sanctis et al., Nature 528, 241-244; 2015).

Dawn achieved many interesting firsts, some of which are the combined result of the spacecraft's design and the innovative operations principles to take advantage of that design. It is the only mission ever to orbit two extraterrestrial destinations, an accomplishment enabled by solar electric propulsion (SEP). SEP has ten times the efficiency of conventional chemical propulsion. Without SEP, a mission to orbit only one of Dawn's targets would have been unaffordable in the Discovery Program, and a mission to orbit both would have been impossible. This innovative system also allowed extensive manoeuvring in orbit to optimize the science return. SEP will be used on NASA's upcoming mission to Psyche and is now routinely considered for other deep space missions as well.

Dawn's sensing instruments included a pair of identical German-contributed cameras with a panchromatic and seven colour filters, a visible $(0.25-1 \mu \mathrm{m})$ and infrared $(1-5 \mu \mathrm{m})$ mapping spectrometer contributed by Italy, a gamma-ray and neutron detector (GRaND), and gravity science obtained through Dawn's telecom subsystem and the Deep Space Network. Global mapping of Vesta and Ceres from multiple altitudes provided an extensive dataset, the largest ever returned for an ice-rich body.

Ceres's surface is globally homogeneous, cratered and dull, except in a handful of

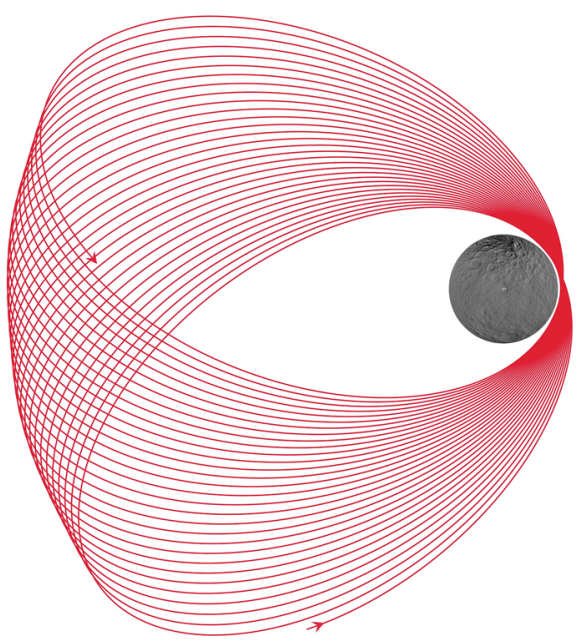

Fig. 1| Representation of Dawn's low elliptical trajectory for a few example orbits. Peridemeter of $\sim 35 \mathrm{~km}$ and apodemeter of $\sim 4,000 \mathrm{~km}$. Credit: NASA/JPL-Caltech/UCLA/MPS/DLR/IDA.

places like Occator, a $\sim 90$-km-diameter crater at $\sim 20^{\circ} \mathrm{N}$ latitude. Highly reflective material in Occator contrasts with the dark surface so strikingly that it was even visible as a bright spot in Hubble images. As Dawn approached Ceres, that one spot turned into two distinct regions: the sharply outlined Cerealia Facula at the crater centre and the Vinalia Faculae, a set of more diffuse deposits to the east. Both areas display a significant amount of sodium carbonate, a marker of warm, alkaline waters. Bright material on an otherwise low-albedo surface should darken rapidly as a result of micrometeorite gardening. Hence, the high albedo of these sites (up to 0.6 at Cerealia) and low crater count indicate their material was emplaced as recently as a few million years ago, much more recently than the $\sim 20$-Mya formation of the crater itself.

Better understanding the source of the sodium carbonate and drivers for that recent exposure became the core motivation for Dawn's second and final extended mission (2017-2018), the results of which are featured in this issue. To increase spatial resolution, the flight team designed a highly elliptical orbit with a peridemeter altitude of $\sim 35 \mathrm{~km}, 11$ times lower than in the prime mission (Fig. 1). Because of the astrobiological interest, Dawn was subject to planetary protection requirements and required to remain on a stable orbit for at least 20 years. That phase presented many challenges, especially with the absence of reaction wheel control following the failures of two wheels in the prime mission and a third in the first extended mission. Hydrazine had been carried only for desaturating the wheels (and some safe modes), but it became a critical resource without wheels. Optimized use of hydrazine enabled the low-altitude orbit to run from June to October 2018, for a total of 127 orbits, and significantly exceeding baseline plans. As the orbit precessed, highresolution observations progressed from Occator crater southward at a rate of $1.9^{\circ}$ per 27-hour revolution. Visible images were returned with a ground sampling as small as $3.3 \mathrm{~m}$ per pixel over part of the faculae and the rest of Occator's floor. GRaND data were acquired with a footprint of $\sim 50 \mathrm{~km}$, and gravity data led to a local gravity field up to degree 45 (versus 18 globally at the end of the prime mission). Infrared spectra and colour images were acquired for selected regions focused on the faculae. The final extended mission's harvest added to Dawn's extensive datasets and represents a fine ending to a grand adventure of exploration and discovery.

Julie C. Castillo-Rogez and Marc D. Rayman (D)

Jet Propulsion Laboratory, California Institute of Technology, Pasadena, CA, USA. e-mail: julie.c.castillo@jpl.nasa.gov; mrayman@jpl.nasa.gov

Published online: 17 March 2020 https://doi.org/10.1038/s41550-020-1031-5

Acknowledgements

This work was carried out at the Jet Propulsion Laboratory, California Institute of Technology, under a contract with the National Aeronautics and Space Administration (80NM0018D0004). 